\title{
A NEW NON-REDUNDANT COMPLEX HILBERT WAVELET TRANSFORMS
}

\author{
Liying Wei and Thierry Blu \\ Department of Electronic Engineering, The Chinese University of Hong Kong, \\ Shatin, N.T., Hong Kong
}

\begin{abstract}
In this paper, a novel non-redundant complex wavelet transform (NRCWT) for real-valued signals is proposed. For this purpose, an orthogonal complex filter bank is developed to implement this NRCWT. We show how to choose the two complex filters from classical real-valued wavelet filters in such a way that the filterbank is always orthogonal. Using fractional B-spline filters, a pair of exact Hilbert wavelets are constructed, which can separate the positive frequencies from the negative frequencies.
\end{abstract}

Index Terms - Non-redundant, complex wavelet transform, multiresolution analysis

\section{INTRODUCTION}

Due to the approximate Hilbert-transform pair of the real part and imaginary part of the wavelet transform coefficients, the dual-tree complex wavelet transform (DTCWT) provides two remarkable advantages over the real wavelet tranform $[1,2]$ : 1) near shift invariance for one-dimensional signals; and 2) angular sensitivity for two-dimensional images. However, this transform is redundant and hence, may not be suitable for such applications as image/video compression where a critically signal representation is required. In order to achieve the non-redundancy for a real-valued signal while having the ability to distinguish positive frequencies from negative frequencies, Spaendonck et.al. [3] developed a new nonredundant complex wavelet transform (NRCWT) based on a three-band orthogonal filter bank where the lowpass filter is real and decimated by "2" and the highpass filters consisting of a common real highphass filter followed by an approximated Hilbert transform filter are decimated by " 4 ". However, only the ideal sinc filters can successfully eliminate the spurious interferences between low and high frequencies. Another NRCWT approach using a triband filter bank [4] might be able to mitigate this issue, but this solution results in a much coarser discrete wavelet decomposition compared to dyadic decompositions.

This work was supported by the General Research Fund CUHK410209 from the Hong Kong Research Grant Council. Email: lywei@ee.cuhk.edu.hk, tblu@ee.cuhk.edu.hk
In this paper, we propose a new non-redundant complex wavelet transform for real-valued input signals based on the structure shown in Fig. 1. Our objective is to show how to choose the complex filters $\{H, G\}$, such that: 1) the filter bank is orthogonal, and 2) it generates an exact Hilbert wavelet, i.e., the frequency response of the wavelet is only supported on either positive frequencies or negative frequencies. For any filter $h[n]$ whose z-tranform corresponds to $H(z)=\sum_{n} h[n] z^{-n}$ (here, $z=e^{j \omega}$ is on the unit circle), $H^{*}(z)=\sum_{n} h^{*}[n] z^{-n}$ where $[\cdot]^{*}$ denotes the conjugation.

\section{NRCWT ORTHOGONAL FILTERBANK}

\subsection{Filterbank structure}

The filterbank in Fig. 1 is the foundation of our non-redundant complex wavelet tranform (NRCWT). Observing the analysis filter bank, the top branche, which correspond to the lowpass channels and consist of two complex lowpass filters $H(z)$ and $H^{*}(z)$ followed by two complex orthonormal filters $U(z)$ and $U^{*}(z)$ and some downsampling/upsampling operators, are summed together to form a real-valued output $y[n]$. This band is a typical multifilter bank branch [5]. The bottom branch corresponds to the highpass channel. The complex wavelet transform is obtained by iterating over the lowpass output. The non-redundancy is obvious: assume $x[n]$ is real and contains $N$ real numbers, then the lowpass output $y[n]$ has $\frac{N}{2}$ real numbers, and the wavelet coefficients $v[n]$ contains $\frac{N}{4}$ complex numbers, so the total storage is $N$.

Here, $U(z)$ and $U^{*}(z)$ are orthonormal filters derived from the Haar filter by frequency shifting,

$$
U(z)=\frac{1+j z^{-1}}{\sqrt{2}}, \quad U^{*}(z)=\frac{1-j z^{-1}}{\sqrt{2}} .
$$

The purpose of $\left\{U(z), U^{*}(z)\right\}$ is to rearrange the output signals from the " $\downarrow 4$ " operators, such that the lowpass output $y[n]$ is real.

\subsection{Complex multiresolution analysis}

Notably, different from other NRCWTs which involve only one scaling function and two wavelets $[3,4]$, our filterbank structure generates two scaling functions $\left\{\phi_{1}(t), \phi_{2}(t)\right\}$ 


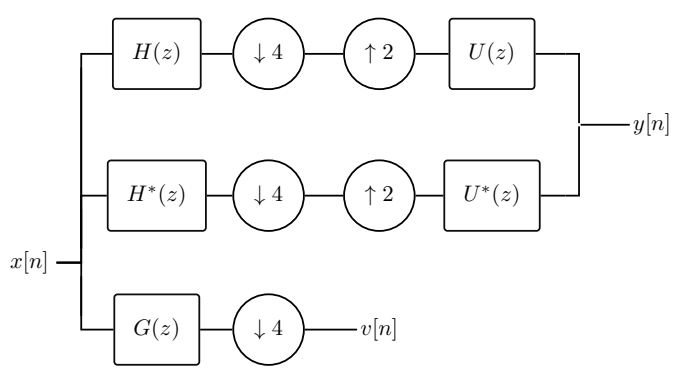

(a) Analysis filter bank

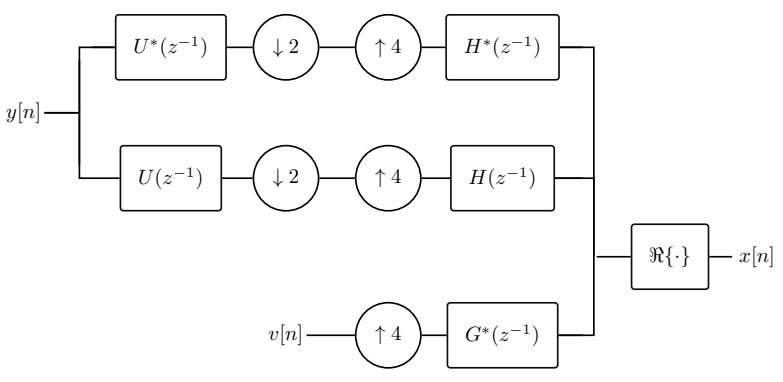

(b) Synthesis filter bank

Fig. 1. Analysis and synthesis orthconjugate filter bank for the non-redundant complex wavelet transform implementation (NRCWT). $y[n]$ is to be iterated for the implementation of CWT. $\Re\{\cdot\}$ is the operator to choose the real part of the summation.

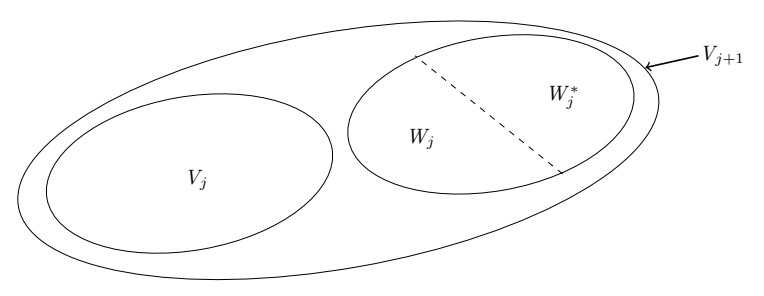

Fig. 2. Geometrical structure of our proposal for the nonredundant complex wavelet multiresolution analysis. $V_{j}$ and $W_{j}$ designate "real" multiresolution spaces, while $W_{j}^{*}$ designates complex multiresolution space.

and two complex conjugate wavelets $\left\{\psi(t), \psi^{*}(t)\right\}$. Both $\left\{\phi_{1}(t), \phi_{2}(t)\right\}$ and $\left\{\psi(t), \psi^{*}(t)\right\}$ form a multiresolution analysis (MRA) that can be understood from multiwavelet framework. Define the scaling space $V_{j}$ and wavelet spaces $W_{j}$ and $W_{j}^{*}(j \in \mathbb{Z})$ as

$$
\begin{aligned}
& V_{j}=\operatorname{Span}_{n \in \mathbb{Z}}\left\{\phi_{1}\left(2^{j} t-2 n\right), \phi_{2}\left(2^{j} t-2 n\right)\right\}, \\
& W_{j}=\operatorname{Span}_{n \in \mathbb{Z}}\left\{\psi\left(2^{j} t-2 n\right)\right\}, \\
& W_{j}^{*}=\operatorname{Span}_{n \in \mathbb{Z}}\left\{\psi^{*}\left(2^{j} t-2 n\right)\right\} .
\end{aligned}
$$

Hence, there exist:

$$
\begin{array}{r}
V_{j} \subset \boldsymbol{L}^{2}(\mathbb{R}), \quad V_{j} \subset V_{j+1}, \\
V_{j} \oplus W_{j} \oplus W_{j}^{*}=V_{j+1}, \\
\forall f(t) \in V_{j} \Longleftrightarrow f\left(2^{-j} t\right) \in V_{0},
\end{array}
$$

where " $\oplus$ " denotes the direct sum. Fig. 2 is the embeddedness graph of the MRA spaces.

Given an input $x[n]$, the lowpass output $y[n]$ can be expressed as

$$
y[n]=\sum_{k, l} 2 \Re\{u[2 k-n] h[l-4 k]\} x[l],
$$

where $\Re\{\cdot\}$ denotes the real-part operator, $u[n]=\frac{\sqrt{2}}{2}(1, j)$, $h[n]$ and $h[n]^{*}$ are the impulse responses of filters $U, H$ and
$H^{*}$, respectively. The highpass output $v[n]$ can be expressed as

$$
v[n]=\sum_{k} g[k-4 n] x[k],
$$

where $g[n]$ is the impulse response of $G(z)$.

Define $\phi_{2 m}(t)=\phi_{1}\left(\frac{t}{2}-m\right)$ and $\phi_{2 m+1}(t)=\phi_{2}\left(\frac{t}{2}-\right.$ $m)$. Then for any square integral function $x(t) \in V_{0}$, its expansion coefficients $x[n]$ can be expressed as $x[m]=$ $\int x(t) \phi_{m}(t)$ and $y[m]=\frac{\sqrt{2}}{2} \int x(t) \phi_{m}\left(\frac{t}{2}\right)$ for $m \in \mathbb{Z}$. Hence, substituting them into (3), we have

$$
\phi_{m}\left(\frac{t}{2}\right)=\sum_{k, l} 2 \Re\{u[2 k-m] h[l-4 k]\} \phi_{l}(t)
$$

Similarly, for the wavelets, we have

$$
\psi_{n}\left(\frac{t}{2}\right)=\frac{\sqrt{2}}{2} \sum_{k} g[k-4 n] \phi_{k}(t) .
$$

In this paper, we aim to design the complex filters $\{H, G\}$, such that the complex conjugate wavelet $\psi(t)$ has only oneside frequency support, which can separate the negative frequencies from the positive frequencies and therefore, completely eliminate spurious interferences between low and high frequencies for practical applications.

\subsection{Orthogonal complex conjugate filters}

In the following, two complex filters are provided such that our proposed filter bank is always orthogonal. The proof will be given in a forthcoming paper.

Theorem 2.1. Assume $\left\{H_{i}, G_{i}\right\}$ for $i=0,1$ consist of a classical standard perfect reconstruction orthogonal two-band filter bank with $H_{i}$ lowpass filter and $G_{i}$ highpass filter, i.e., they must satisfy

$$
\begin{array}{r}
H_{i}(z) H_{i}\left(z^{-1}\right)+G_{i}(z) G_{i}\left(z^{-1}\right)=2, \\
H_{i}(-z) H_{i}\left(z^{-1}\right)+G_{i}(-z) G_{i}\left(z^{-1}\right)=0, \\
G_{i}(z)=-z^{-1} H\left(-z^{-1}\right) .
\end{array}
$$


Hence, by choosing

$$
\begin{aligned}
H(z) & =\frac{\sqrt{2}}{2}\left[H_{0}\left(z^{2}\right)-j z^{-1} H_{1}\left(z^{2}\right)\right], \\
H^{*}(z) & =\frac{\sqrt{2}}{2}\left[H_{0}\left(z^{2}\right)+j z^{-1} H_{1}\left(z^{2}\right)\right], \\
G(z) & =\frac{\sqrt{2}}{2}\left[G_{0}\left(z^{2}\right)-j z^{-1} G_{1}\left(z^{2}\right)\right],
\end{aligned}
$$

then $\{H(z), G(z)\}$ forms a perfect reconstruction orthogonal filter bank for the filterbank structure as shown in Fig. 1.

Our objective is now to construct a wavelet which is only supported either on positive frequencies or negative frequencies. Using wavelet theory, it is equivalent to construct a highpass filter whose frequency support is one-sided. Currently, Selesnick [6] has developed a procedure to design an approximate Hilbert transform pairs of wavelet bases based on spectral factorization. Chaudhury and Unser [7] constructed another Hilbert transform pairs of wavelet bases based on fractional B-spline functions [8]. In this paper, we use the orthonormal fractional $(\alpha, \tau)$-spline filters to construct our Hilbert wavelets $\psi(t)$ and $\psi^{*}(t)$.

According to Theorem 2.1, we choose

$$
H_{0}(z)=H_{0, \perp}^{\alpha}(z), H_{1}(z)=H_{1 / 2, \perp}^{\alpha}(z),
$$

where $H_{\tau, \perp}^{\alpha}$ is the orthonormal fractional $(\alpha, \tau)$-spline filters with degree $\alpha>-\frac{1}{2}$ and shift $\tau$,

$$
\begin{aligned}
& H_{\tau, \perp}^{\alpha}\left(e^{j \frac{\omega}{2}}\right)=H_{\tau}^{\alpha}\left(e^{j \frac{\omega}{2}}\right) \sqrt{\frac{A^{\alpha}\left(e^{j \frac{\omega}{2}}\right)}{A^{\alpha}\left(e^{j \omega}\right)}}, \\
& H_{\tau}^{\alpha}\left(e^{j \omega}\right)=2^{-(\alpha+1)}\left(1+e^{j \omega}\right)^{\frac{\alpha+1}{2}-\tau}\left(1+e^{-j \omega}\right)^{\frac{\alpha+1}{2}+\tau}, \\
& A^{\alpha}\left(e^{j \omega}\right)=\sum_{k}\left|\widehat{\beta}_{0}^{\alpha}(\omega+2 k \pi)\right|^{2}, \\
& \widehat{\beta}_{\tau}^{\alpha}(\omega)=\left(\frac{1-e^{-j \omega}}{j \omega}\right)^{\frac{\alpha+1}{2}+\tau}\left(\frac{e^{j \omega}-1}{j \omega}\right)^{\frac{\alpha+1}{2}-\tau},
\end{aligned}
$$

where $A^{\alpha}\left(e^{j \omega}\right)$ is the autocorrelation function of the fractional B-spline functions $\widehat{\beta}_{\tau}^{\alpha}(\omega)$. Because $\tau$ only affects the phase of the filter, hence, $\left|H_{0, \perp}^{\alpha}\left(e^{j \omega}\right)\right|=\left|H_{1 / 2, \perp}^{\alpha}\left(e^{j \omega}\right)\right|$ for any $\alpha>\frac{1}{2}$. Fig. 3 depicts the magnitudes of the Fourier responses of the filters $\left\{H\left(e^{j \omega}\right), G\left(e^{j \omega}\right)\right\}$ obtained using Theorem 2.1. Obviously, $\left.\mid G\left(e^{j \omega}\right)\right\} \mid$ is only supported on the negative frequencies, which implies $\mid G^{*}\left(e^{j \omega} \mid\right.$ is only supported on the positive frequencies. We will see that the associated wavelets also have a one-sided frequency support.

Given the special filters $H_{0}(z)$ and $H_{1}(z)$ (9), according to Theorem 2.1, we get $H$ and $G$. Then substituting $H$ into (5), we solve the scaling functions which in the frequency domain can be expressed as

$$
\widehat{\phi}_{1}(\omega)=\widehat{\beta}_{0, \perp}^{\alpha}(\omega), \quad \widehat{\phi}_{2}(\omega)=\widehat{\beta}_{1 / 2, \perp}^{\alpha}(\omega),
$$
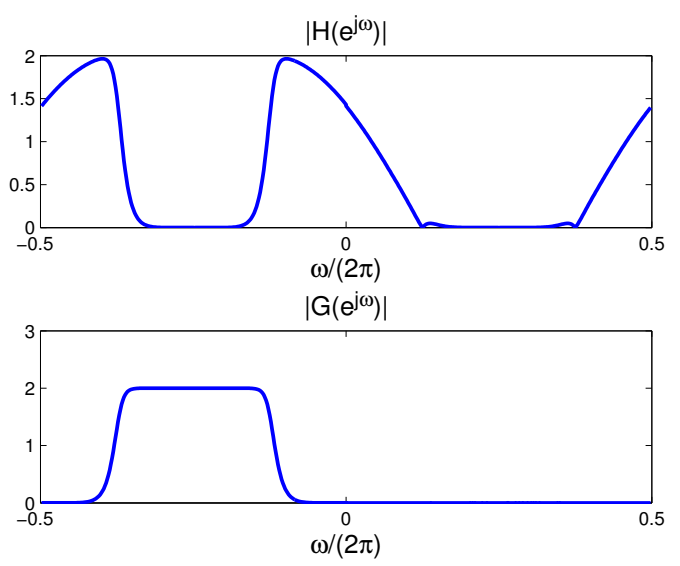

Fig. 3. The magnitudes of Fourier responses $\{H, G\}$ when the fractional B-spline filters for $\alpha=4.5$ are used.

where the orthonormalized fractional B-spline functions $\widehat{\beta}_{\tau, \perp}^{\alpha}(\omega)=\frac{\widehat{\beta}_{\tau}^{\alpha}(\omega)}{\sqrt{A^{\alpha}\left(e^{j \omega}\right)}}$. Fig. 4 displays the orthonormalized fractional B-spline functions $\beta_{\tau, \perp}^{\alpha}(t)$ for $\alpha=4.5$ and $\tau=0,1 / 2$, respectively. It is clear that: 1) $\beta_{\tau, \perp}^{\alpha}(t)$ is well localized, and 2) $\beta_{0, \perp}^{4.5}(t)$ and $\beta_{1 / 2, \perp}^{4.5}(t)$ have the same waveform except the shift difference, which again demonstrates $\tau$ only affects the phase.
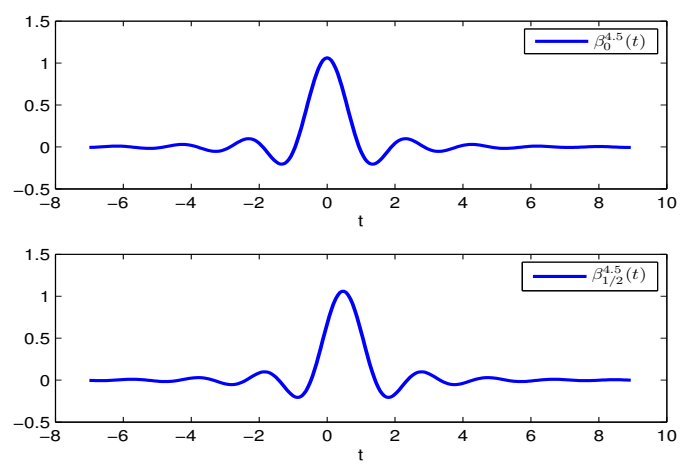

Fig. 4. Plots of $\beta_{\tau, \perp}^{\alpha}(t)$ for $\alpha=4.5$ and $\tau=0,1 / 2$.

Substituting $G$ into (6) and using the two-scale relation of the orthonormalized fractional B-spline functions,

$$
\widehat{\beta}_{\tau, \perp}^{\alpha}(\omega)=\frac{\sqrt{2}}{2} H_{\tau, \perp}^{\alpha}\left(e^{j \omega}\right) \widehat{\beta}_{\tau, \perp}^{\alpha}\left(\frac{\omega}{2}\right) .
$$

the wavelets can be expressed as

$$
\begin{array}{r}
\widehat{\psi}(\omega)=\frac{1}{2} \widehat{\beta}_{0, \perp}^{\alpha}\left(\frac{\omega}{2}\right) G_{0, \perp}^{\alpha}\left(e^{j \frac{\omega}{2}}\right)[1-\operatorname{sgn}(\omega)], \\
\widehat{\psi}^{*}(-\omega)=\frac{\sqrt{2}}{4} \widehat{\beta}_{0, \perp}^{\alpha}\left(\frac{\omega}{2}\right) G_{0, \perp}^{\alpha}\left(e^{j \frac{\omega}{2}}\right)[1+\operatorname{sgn}(\omega)],
\end{array}
$$


where $G_{0, \perp}^{\alpha}\left(e^{j \omega}\right)=e^{-j \omega} H_{0, \perp}^{\alpha}\left(-e^{-j \omega}\right)$. It is also easy to deduce that the wavelets are not orthogonal themselves and also not orthogonal to the scaling functions. Surprisingly, our construction leads to a orthonormal multifilter bank, but the MRA is made of function space that are not orthogonal. Fig. 5 plots
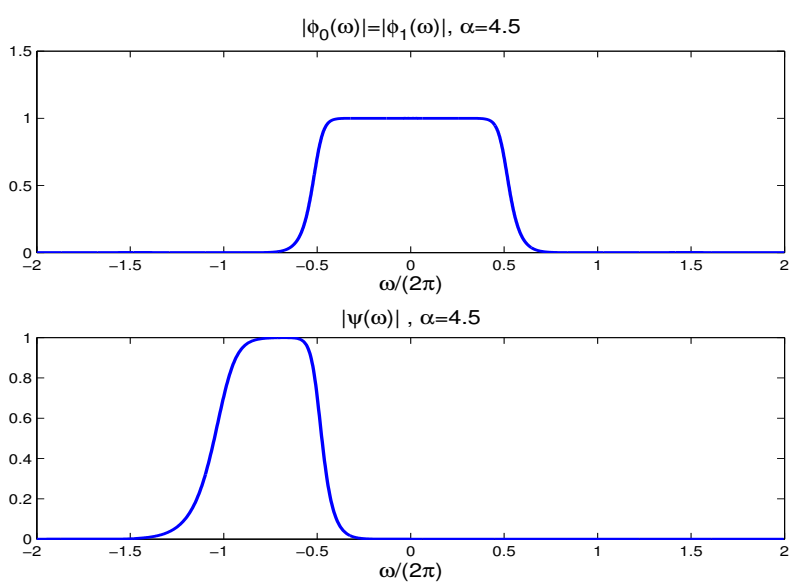

Fig. 5. The magnitudes of the scaling function $\widehat{\phi}_{1,2}(\omega)$ and the wavelet $\widehat{\psi}_{1}(\omega)$ when the fractional B-spline filters for $\alpha=$ 4.5 are used. In this figure, the real parts and imaginary parts of $\widehat{\phi}_{1,2}(\omega)$ are also plotted.

the magnitude of the Fourier responses of the scaling function $\widehat{\phi}_{1}(\omega)$ and the wavelet $\widehat{\psi}_{1}(\omega)$. The figure shows that: 1$)$ $\left|\widehat{\phi}_{1}(\omega)\right|$ is symmetric, 2) the frequency support of $\left|\widehat{\phi}_{1,2}(\omega)\right|$ is greater than $[-\pi, \pi]$, which implies that the filters are not ideal, and 3) the wavelet is supported on the negative frequencies.

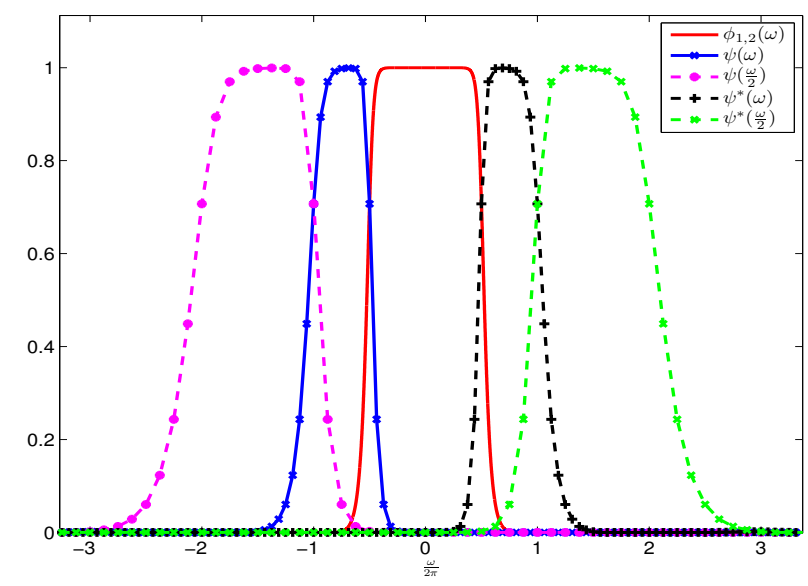

Fig. 6. The magnitudes of Fourier responses of the scaling functions and the wavelets at different scales when the fractional B-spline filters for $\alpha=4.5$ are used.

Fig. 6 plots the magnitudes of Fourier responses for scaling functions in $V_{0}$ and wavelets in $W_{0}$ and $W_{1}$ when the fractional B-spline filters are used. The middle one is the scaling function, and the left and right are the wavelets. It is clear that supports of the wavelets and scaling functions are overlapped. Hence, the wavelets are not orthogonal to the scaling functions.

\section{CONCLUSION}

In this paper, we proposed a new non-redundant complex wavelet transform by using an orthogonal two-band multifilter bank, and constructed a pair of Hilbert wavelets by using fractional spline filters. Our future work is to apply our framework in image denoising and compression.

\section{REFERENCES}

[1] N. Kingsbury, "Image processing with complex wavelets," Phil. Trans. R. Soc. Lond. A, vol. 357, no. 1760, pp. 2543-2560, Sept. 1999.

[2] I. W. Selesnick, R. G. Baraniuk, and N. G. Kingsbury, "The dual-tree complex wavelet transform," IEEE Signal Process. Mag., vol. 22, no. 6, pp. 123-151, Nov. 2005.

[3] R. V. Spaendonck, T. Blu, R. Baraniuk, and M. Vetterli, "Orthogonal Hilbert transform filter banks and wavelets," in Proc. IEEE Int. Conf. Acoust., Speech, Signal Processing (ICASSP'2003), Hong Kong, China, April 6-10 2003, pp. 505-508.

[4] F. C. A. Fernandes, , M. B. Wakin, and R. G. Baraniuk, "Non-redundant, linear-phase, semi-orthogonal, directional complex wavelets," in Proc. IEEE Int. Conf. Acoust., Speech, Signal Processing (ICASSP'2004), Montreal, Quebec, Canada, May 2004, pp. 953-956.

[5] Xiang-Gen Xia and B. W. Suter, "Vector-valued wavelets and vector filter banks," IEEE Trans. Signal Process., vol. 44, no. 3, pp. 508-518, Mar. 1996.

[6] I. W. Selesnick, "The design of approximate Hilbert transform pairs of wavelet bases," IEEE Trans. Signal Process., vol. 50, no. 5, pp. 1144-1152, May 2002.

[7] K. N. Chaudhury and M. Unser, "Construction of Hilbert transform pair of wavelet bases and Gabor-like transforms," IEEE Trans. Sign. Process., vol. 57, no. 9, pp. 3411-3425, Sept. 2009.

[8] T. Blu and M. Unser, "A complete family of scaling functions: the $(\alpha, \tau)$-fractional splines," in Proc. IEEE Int. Conf. Acoust., Speech, Signal Processing (ICASSP'2003), Hong Kong, People's Republic of China, April 2003, pp. 421-424. 